\title{
Data Visualization + Scrollytelling for Election News Stories : Challenges and Perspectives
}

\author{
Jean-Philippe Corbeil* \\ Le Devoir
}

\author{
Florent Daudens ${ }^{\dagger}$ \\ Le Devoir
}

\author{
Thomas Hurtut \\ Polytechnique Montreal
}

\begin{abstract}
This visual case study is conducted by Le Devoir, a Canadian frenchlanguage independent daily newspaper gathering around 50 journalists and one million readers every week. During the past twelve months, in collaboration with Polytechnique Montreal, we investigated a scrollytelling format strongly relying on combined series of data visualizations. This visual case study will specifically present one of the news stories we published, which communicates electoral results the day after the last Quebec general election. It gathers all the lessons that we learnt from this experience, the challenges that we tackled and the perspectives for the future. Beyond the specific electoral context of this work, these conclusions might be useful for any practioner willing to communicate data visualization based stories, using a scrollytelling narrative format.
\end{abstract}

Index Terms: Human-centered computing_-Visualization-Visualization techniques

\section{Case study}

We present a case study of a news story we published at Le Devoir for the last Quebec general election. It is available online at https://www. ledevoir.com/documents/special/18-10_ resultats_election_qc_2018/index.html. It investigates a format based on scrollytelling and a heavy use of visualizations, ordered meaningfully, as defined in [3]. It is an effective and powerful narrative format to package and transmit complex information [2,5]. Yet, it raises many challenges in the production environment of a press room as we will detail in this section.

General Description The day after the last Quebec general election, a political party called CAQ surprised the population and analysts by its massive victory, defying the polls. We wanted to quickly release a visualization based story in order to give insights on this remarkable result. Following Munzer's data abstraction [4], we had in hand the electoral results for each of the 125 district and its running political parties. In terms of task and design, given the extreme size heterogeneity of the Quebec districts, we presented the spatial correlation over the whole province using a unit-based map. The singularity of this election also incited us to compare these results to the 2014 election both for the districts' winning parties, and for their vote margins, using a pyramid based design.

Design Considerations Nowadays, around $60 \%$ of the online readers of Le Devoir are on mobile devices. Besides, it is easier to adapt a data visualization from a small screen dimension to a larger one, than vice versa. Thus, the design process mantra we try to instill is mobile first, then desktop. In mass media, data visualization literacy is also limited. Readers need guidance and clarity. A crucial effort and attention is to gradually deliver an

*e-mail: jpcorbeil@ledevoir.com

†e-mail: fdaudens@ledevoir.com

$\ddagger$ e-mail: thomas.hurtut@polymtl.ca even slightly above the average visualization in terms of complexity. In that perspective, scrollytelling and transitions bring powerful supports to help the reader understand the chosen design, and avoid to lead him astray [1].

These principles are used several times in our work for instance by introducing the unit-based map of the electoral results using three progressive steps. Thoughtful transitions between different designs for the same data are also implemented, such as the one between the map and the seating plan of the national assembly members. The rather original pyramid visualization at the end is also gradually introduced in several steps.

Technical Issues Design must be responsive in order to adapt to all possible ratios and dimensions. This requires to resize the visualizations without corrupting their content, possibly on a vertical orientation and a much more limited screen space. Similarly to the constraining mobile/desktop mantra, tests are made on lowperformance devices.

Interdisciplinarity Challenges The use of visualization in online news stories embed the work of journalists, software engineers, designers and even sometimes data scientists. Added to time constraints and the tempo of news production, operating such interdisciplinarity teamwork require ingenuity, planning (for instance by using story-boards and post-mortem meetings) and good communication. These different people must found a common basis to communicate and be eager to learn about, and from, each others.

Although many challenges need to be tackled, we observed that this kind of narrative format provides several important benefits. First it brings innovative journalism a step further allowing a deeper analysis of the data behind the story. It also helped Le Devoir to distinguish itself from its competitors. Besides, we observed that the stories we produced using this format regularly hit our most-read articles in terms of audience and reader retention.

\section{ONGOING AND FUtURE WORK}

We are presently building a new scrollytelling template allowing the combination of a more diverse type of content (long text, video, sound, animation). Automation is crucial for a midsize press room such as ours. We investigate approaches to abstract the decomposition of complex data visualizations into progressive user friendly steps. Lastly, interaction is a challenging remaining issue, constrained both by design and technical aspects but having a great potential to explore.

\section{REFERENCES}

[1] A. Cairo. How charts lie. W. W. Norton \& Company, 2019.

[2] R. Kosara and J. Mackinlay. Storytelling: The next step for visualization. Computer, 46(5), 2013.

[3] B. Lee, N. H. Riche, P. Isenberg, and S. Carpendale. More than telling a story: Transforming data into visually shared stories. IEEE Computer Graphics \& Applications, 35(5), 2015.

[4] T. Munzner. Visualization analysis and design. AK Peters/CRC Press, 2014.

[5] D. Seyser and M. Zeiller. Scrollytelling - an analysis of visual storytelling in online journalism. In Proc. Int. Conf. Inf. Vis. (IV), 2018. 